\title{
3-Total Edge Sum Cordial Labeling for Some Graphs
}

\author{
Abha Tenguria \\ Department of Mathematics, \\ Govt. MLB P.G. Girls Autonomus College, Bhopal
}

\author{
Rinku Verma \\ Department of Mathematics, \\ Medicaps Institute of Science and Technology, Indore
}

\begin{abstract}
The sum cordial labeling is a variant of cordial labeling. Here a variant of 3-total sum cordial labeling was introduced and name it as 3-total edge sum cordial labeling unlike in 3-total sum cordial labeling the roles of vertices and edges are interchanged. Here in this paper path graph, cycle graph and complete bipartite graph $k_{1}, n$ are investigated on this newly defined concept.
\end{abstract}

\section{General Terms}

2000 AMS Subject Classification: 05C78

\section{Keywords}

Cordial labeling, Edge sum cordial labeling, 3-Total edge sum cordial labeling, 3-Total edge sum cordial graphs

\section{INTRODUCTION}

The graphs consider here are simple, finite, connected and undirected graphs for all other terminology and notation follow Harray [3]. Let $G(V, E)$ be a graph where the symbols $V(G)$ and $E(G)$ denotes the vertex set and edge set. If the vertices or edges or both of the graph are assigned values subject to certain conditions it is known as graph labeling. A dynamic survey of graph labeling is regularly updated by Gallian [2] and it is published in Electronic Journal of Combinatorics. Cordial graphs was first introduced by Cahit [1] as a weaker version of both graceful graphs and harmonious graphs. The concept of sum cordial labeling of graph was introduced by Shiama J. [4]. The concept of 3-Total super sum cordial labeling of graphs was introduced by Tenguria Abha and Verma Rinku [5]. The concept of 3-Total super product cordial labeling of graphs was introduced by Tenguria Abha and Verma Rinku [6]. Edge product cordial labeling of graphs was introduced by S. K. Vaidya and C. M. Barasara [8]. Here brief summary of definitions are given which are useful for the present investigations.

DEFINITION 1. Let $G$ be a graph. Let $f$ be a map from $V(G)$ to $\{0,1,2\}$. For each edge uv assign the label $[f(u)+$ $f(v)](\bmod 3)$. Then the map $f$ is called 3-total sum cordial labeling of $G$, if $|f(i)-f(j)| \leq 1 ; i, j \in\{0,1,2\}$ where $f(x)$ denotes the total number of vertices and edges labeled with $x=\{0,1,2\}$.

In this paper we introduce the edge analogue of 3-total sum cordial labeling and investigate the results for some standard graphs.

DEFINITION 2. For graph $G$ the edge labeling function is defined as $f: E(G) \rightarrow\{0,1,2\}$ and induced vertex labeling func- tion $f^{*}: V(G) \rightarrow\{0,1,2\}$ is given as if $e_{1}, e_{2}, \ldots, e_{n}$ are the edges incident to vertex $v$ then $f^{*}(v)=f\left(e_{1}\right)+{ }_{3} f\left(e_{2}\right)+{ }_{3} \ldots+{ }_{3}$ $f\left(e_{n}\right)$. Then the map $f$ is called 3 -total edge sum cordial labeling of a graph $G$ if $|f(i)-f(j)| \leq 1 ; i, j \in\{0,1,2\}$ where $f(x)$ denotes the total number of vertices and edges labeled with $x=\{0,1,2\}$.

\section{MAIN RESULTS}

THEOREM 3. The path graph $P_{n}$ is 3-total edge sum cordial.

Proof: Let $e_{1}, e_{2}, \ldots, e_{n-1}$ be edges of path $P_{n}$

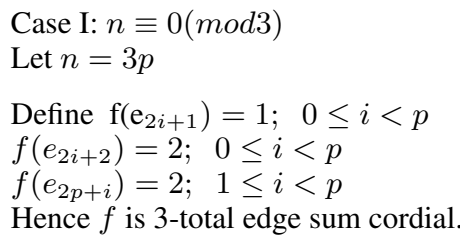

Case II: $n \equiv 1(\bmod 3)$

Let $n=3 p+1$

Assign

$\mathrm{f}\left(\mathrm{e}_{n-1}\right)=0$

$f\left(e_{n-2}\right)=1$

$f\left(e_{n-3}\right)=2$

Define

$\mathrm{f}\left(\mathrm{e}_{3 i+1}\right)=2 ; \quad 0 \leq i<p-1$

$f\left(e_{3 i+2}\right)=2 ; \quad 0 \leq i<p-1$

$f\left(e_{3 i+3}\right)=1 ; 0 \leq i<p-1$

Hence $f$ is 3 -total edge sum cordial.

Case III: $n \equiv 2(\bmod 3)$

Let $n=3 p+2$

Define

$\mathrm{f}\left(\mathrm{e}_{3 i+1}\right)=2 ; \quad 0 \leq i<p-1$

$f\left(e_{3 i+2}\right)=2 ; \quad 0 \leq i<p-1$

$f\left(e_{3 i+3}\right)=1 ; \quad 0 \leq i<p-1$

Assign
$\mathrm{f}\left(\mathrm{e}_{n-1}\right)=1$
$f\left(e_{n-2}\right)=f\left(e_{n-4}\right)=2$
$f\left(e_{n-3}\right)=1$

Hence $f$ is 3-total edge sum cordial. 
THEOREM 4. The cycle graph $C_{n}$ is 3-total edge sum cordial.

Proof: Let $e_{1}, e_{2}, \ldots, e_{n}$ be the edges of cycle $C_{n}$

Case I: $n \equiv 0(\bmod 3)$

Let $n=3 p$

Define

$\mathrm{f}\left(\mathrm{e}_{3 i+1}\right)=2 ; \quad 0 \leq i \leq p-1$

$f\left(e_{3 i+2}\right)=2 ; \quad 0<i<p-1$

$f\left(e_{3 i+3}\right)=1 ; 0 \leq i \leq p-1$

Hence $f$ is 3 -total edge sum cordial.

Case II: $n \equiv 1(\bmod 3)$

Let $n=3 p+1$

Assign

$\mathrm{f}\left(\mathrm{e}_{1}\right)=f\left(e_{3}\right)=f\left(e_{4}\right)=1$

$f\left(e_{2}\right)=2$

Define

$\mathrm{f}\left(\mathrm{e}_{3 i+5}\right)=2 ; \quad 0 \leq i<p-1$

$f\left(e_{3 i+6}\right)=1 ; \quad 0 \leq i<p-1$

$f\left(e_{3 i+7}\right)=1 ; 0 \leq i<p-1$

Hence $f$ is 3 -total edge sum cordial.

Case III: $n \equiv 2(\bmod 3)$

Let $n=3 p+2$

Assign

$\mathrm{f}\left(\mathrm{e}_{1}\right)=1$

$f\left(e_{2}\right)=2$

Define

$\mathrm{f}\left(\mathrm{e}_{3 i+3}\right)=1 ; \quad 0 \leq i<p$

$f\left(e_{3 i+4}\right)=1 ; 0 \leq i<p$

$f\left(e_{3 i+5}\right)=2 ; 0 \leq i<p$

Hence $f$ is 3 -total edge sum cordial.

THEOREM 5. The complete Bipartite graph $k_{1}, n$ is 3-total edge sum cordial if $n \equiv 0(\bmod 3)$ and $n \equiv 2(\bmod 3)$.

Proof: Let $V\left(k_{1}, n\right)=\left\{v, v_{i} ; 1 \leq i \leq n\right\}$

and $E\left(k_{1}, n\right)=\left\{v v_{i} ; 1 \leq i \leq n\right\}$

Case I: $n \equiv 0(\bmod 3)$

Let $n=3 p$

Define

$\mathrm{f}\left(\mathrm{e}_{3 i+1}\right)=0 ; \quad 0 \leq i<p$

$f\left(e_{3 i+2}\right)=1 ; 0 \leq i<p$

$f\left(e_{3 i+3}\right)=2 ; 0 \leq i<p$

Hence $f$ is 3-total edge sum cordial.

Case II: $n \equiv 2(\bmod 3)$

Let $n=3 p+2$

Define

$\mathrm{f}\left(\mathrm{e}_{3 i+1}\right)=0 ; \quad 0 \leq i<p$

$f\left(e_{3 i+2}\right)=1 ; 0 \leq i \leq p$

$f\left(e_{3 i+3}\right)=2 ; 0 \leq i \leq p$

If $p=0$
Assign

$\mathrm{f}\left(\mathrm{e}_{1}\right)=1$

$f\left(e_{2}\right)=2$

Hence $f$ is 3 -total edge sum cordial.

EXAMPLE 1. The path graph $P_{14}$ is 3-total edge sum cordial.

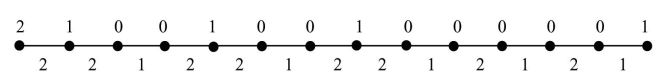

Fig $1: 3$-total edge sum cordial labeling of $P_{14}$

Table 1: Edge and Vertex conditions for 3-Total edge sum cordial labeling of $P_{n}$

\begin{tabular}{|c|c|c|c|}
\hline Case & Edge Condition & Vertex Condition & $f(i)=v_{f}(i)+e_{f}(i)$ \\
\hline$n=3 p$ & $\begin{array}{l}e_{f}(0)=0 \\
e_{f}(1)=p \\
e_{f}(2)=2 p-1\end{array}$ & $\begin{array}{l}v_{f}(0)=2 p-1 \\
v_{f}(1)=p \\
v_{f}(2)=1\end{array}$ & $\begin{array}{l}f(0)=2 p-1 \\
f(1)=2 p \\
f(2)=2 p\end{array}$ \\
\hline$n=3 p+1$ & $\begin{array}{l}e_{f}(0)=1 \\
e_{f}(1)=p \\
e_{f}(2)=2 p-1\end{array}$ & $\begin{array}{l}v_{f}(0)=2 p \\
v_{f}(1)=p \\
v_{f}(2)=1\end{array}$ & $\begin{array}{l}f(0)=2 p+1 \\
f(1)=2 p \\
f(2)=2 p\end{array}$ \\
\hline$n=3 p+2$ & $\begin{array}{l}e_{f}(0)=0 \\
e_{f}(1)=p+1 \\
e_{f}(2)=2 p\end{array}$ & $\begin{array}{l}v_{f}(0)=2 p+1 \\
v_{f}(1)=p \\
v_{f}(2)=1\end{array}$ & $\begin{array}{l}f(0)=2 p+1 \\
f(1)=2 p+1 \\
f(2)=2 p+1\end{array}$ \\
\hline
\end{tabular}

EXAMPLE 2. The cycle graph $C_{5}$ is 3-total edge sum cordial.

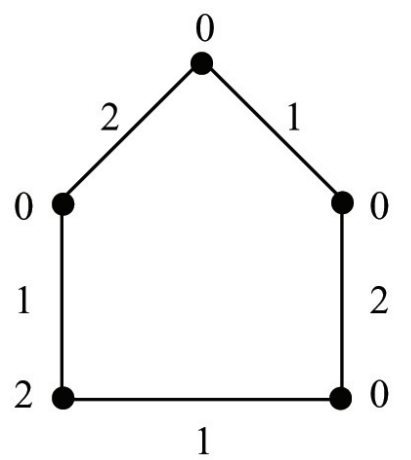

Fig 2:3-total edge sum cordial labeling of $C_{5}$

Table 2: Edge and Vertex conditions for 3-Total edge and sum cordial labeling of $C_{n}$

\begin{tabular}{|l|l|l|l|}
\hline Case & Edge Condition & Vertex Condition & $\boldsymbol{f}(\boldsymbol{i})=\boldsymbol{v}_{\boldsymbol{f}}(\boldsymbol{i})+\boldsymbol{e}_{\boldsymbol{f}}(\boldsymbol{i})$ \\
\hline $\mathrm{n}=3 \mathrm{p}$ & $e_{f}(0)=0$ & $v_{f}(0)=2 p$ & $f(0)=2 p$ \\
& $e_{f}(1)=p$ & $v_{f}(1)=p$ & $f(1)=2 p$ \\
& $e_{f}(2)=2 p$ & $v_{f}(2)=0$ & $f(2)=2 p$ \\
\hline $\mathrm{n}=3 \mathrm{p}+1$ & $e_{f}(0)=0$ & $v_{f}(0)=2 p$ & $f(0)=2 p$ \\
& $e_{f}(1)=2 p+1$ & $v_{f}(1)=0$ & $f(1)=2 p+1$ \\
& $e_{f}(2)=p$ & $v_{f}(2)=p+1$ & $f(2)=2 p+1$ \\
& & & \\
\hline $\mathrm{n}=3 \mathrm{p}+2$ & $e_{f}(0)=0$ & $v_{f}(0)=2 p+2$ & $f(0)=2 p+2$ \\
& $e_{f}(1)=2 p+1$ & $v_{f}(1)=0$ & $f(1)=2 p+1$ \\
& $e_{f}(2)=p+1$ & $v_{f}(2)=p$ & $f(2)=2 p+1$ \\
& & & \\
\hline
\end{tabular}


EXAMPLE 3. The complete Bipartite graph $k_{1,6}$ is 3-total edge sum cordial.

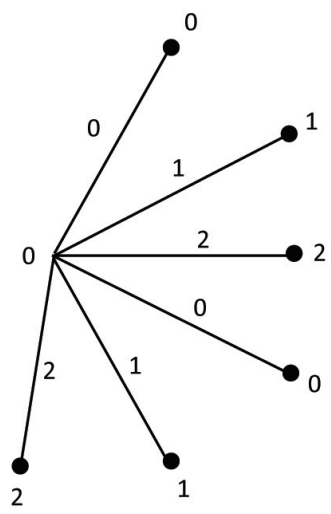

Fig $3: 3$-total edge sum cordial labeling of $k_{1,6}$

Table 3: Edge and Vertex conditions for 3-Total edge and sum cordial labeling of $k_{1}, n$

\begin{tabular}{|l|l|l|l|}
\hline Case & Edge Condition & Vertex Condition & $\boldsymbol{f}(\boldsymbol{i})=v_{\boldsymbol{f}}(\boldsymbol{i})+\boldsymbol{e}_{\boldsymbol{f}}(\boldsymbol{i})$ \\
\hline $\mathrm{n}=3 \mathrm{p}$ & $e_{f}(0)=p$ & $v_{f}(0)=p+1$ & $f(0)=2 p+1$ \\
& $e_{f}(1)=p$ & $v_{f}(1)=p$ & $f(1)=2 p$ \\
& $e_{f}(2)=p$ & $v_{f}(2)=p$ & $f(2)=2 p$ \\
& & & \\
\hline $\mathrm{n}=3 \mathrm{p}+2$ & $e_{f}(0)=p$ & $v_{f}(0)=p+1$ & $f(0)=2 p+1$ \\
& $e_{f}(1)=p+1$ & $v_{f}(1)=p+1$ & $f(1)=2 p+2$ \\
& $e_{f}(2)=p+1$ & $v_{f}(2)=p+1$ & $f(2)=2 p+2$ \\
& & & \\
\hline
\end{tabular}

\section{COROLLARY}

If $G$ is 3 edge sum cordial graph then it is 3 -total edge sum cordial labeling of graph.

\section{CONCLUSION}

Labeling of discrete structure is a potential area of research. We have investigated 3-Total edge sum cordial labeling of graphs to investigate analogous results for different graphs as well in the context of various graph labeling problems is an open area of research.

\section{REFERENCES}

[1] Cahit I., "Cordial graphs: A weaker version of graceful and harmonious graphs" Ars combinatorial 23, 201-207, (1987).

[2] Gallian J. A., "A dynamic survey of graph labeling", The Electronics journal of Combinatorics, 17, (2010) DS6.

[3] Harrary F., Graph theory, Narosa Publishing House, (2001).

[4] Shiama J., "Sum cordial labeling for some graphs", IJMA3(a), 3271-3276, sept-(2012).

[5] Tenguria Abha and Verma Rinku, "3-Total super sum cordial labeling for some graphs" IJMA, 5 (12), 117-121, (2014).
[6] Tenguria Abha and Verma Rinku, "3-Total super product cordial labeling for some graphs" International Journal of Science and Research - 4(2), 557-559, February (2015).

[7] Sundaram M., Ponraj R. and Somasundaram S.," Product cordial labeling of graphs", Bull. Pure and Applied Sciences (Mathematics and Statistics) 23E 155-163 (2004).

[8] Vaidya S. K. and Barasara C. M., "Edge product cordial labeling of graphs", J. Math Comput. Sci. 2(5), 1436-1450, (2012). 